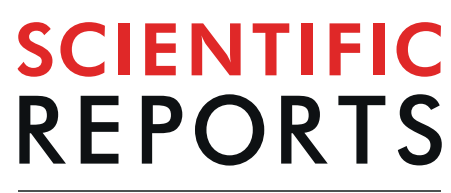

natureresearch

Check for updates

\title{
Inhibition of mitochondrial complex I by the novel compound FSL0260 enhances high salinity-stress tolerance in Arabidopsis thaliana
}

\author{
Kaori Sako $\mathbb{1}^{1,2,9 凶}$, Yushi Futamura $\mathbb{1}^{3}$, Takeshi Shimizu ${ }^{3}$, Akihiro Matsui, ${ }^{1,10}$, \\ Hiroyuki Hirano $\mathbb{D}^{4}$, Yasumitsu Kondoh ${ }^{3}$, Makoto Muroi ${ }^{3}$, Harumi Aono ${ }^{3}$, Maho Tanaka ${ }^{1}$, \\ Kaori Honda ${ }^{3}$, Kenshirou Shimizu ${ }^{3}$, Makoto Kawatani ${ }^{3}$, Takeshi Nakano ${ }^{5,6}$, Hiroyuki Osada $\rrbracket^{3,4}$, \\ Ko Noguchi ${ }^{7}{ }^{7}$ \& Motoaki Seki ${ }^{1,8,9,10 凶}$
}

Chemical priming is an attractive and promising approach to improve abiotic stress tolerance in a broad variety of plant species. We screened the RIKEN Natural Products Depository (NPDepo) chemical library and identified a novel compound, FSL0260, enhancing salinity-stress tolerance in Arabidopsis thaliana and rice. Through transcriptome analysis using A. thaliana seedlings, treatment of FSL0260 elevated an alternative respiration pathway in mitochondria that modulates accumulation of reactive oxygen species (ROS). From comparison analysis, we realized that the alternative respiration pathway was induced by treatment of known mitochondrial inhibitors. We confirmed that known inhibitors of mitochondrial complex I, such as rotenone and piericidin A, also enhanced salt-stress tolerance in Arabidopsis. We demonstrated that FSL0260 binds to complex I of the mitochondrial electron transport chain and inhibits its activity, suggesting that inhibition of mitochondrial complex I activates an alternative respiration pathway resulting in reduction of ROS accumulation and enhancement of tolerance to salinity in plants. Furthermore, FSL0260 preferentially inhibited plant mitochondrial complex I rather than a mammalian complex, implying that FSL0260 has a potential to be an agent for improving salt-stress tolerance in agriculture that is low toxicity to humans.

High salinity stress is one of the major abiotic factors limiting crop productivity. Globally, more than $20 \%$ of irrigated lands are affected by soil salinization ${ }^{1}$. Improving crop salinity-stress tolerance is essential for sustainable food production. High salinity stress induces osmotic stress and ionic stress that inhibit water uptake in roots and photosynthesis in shoots ${ }^{2}$. It also enhances the generation of reactive oxygen species (ROS) such as superoxide anion $\left(\mathrm{O}_{2}{ }^{-}\right)$, hydrogen peroxide $\left(\mathrm{H}_{2} \mathrm{O}_{2}\right)$ and the hydroxyl radical $(\cdot \mathrm{OH})$. ROS works as a signaling molecule; however, excess ROS is highly toxic and leads to metabolic disorders, cell damage and cell death ${ }^{3-5}$. Salinity stress causes over-reduction of the mitochondrial electron transport chain (mETC), which induces electron leakage to $\mathrm{O}_{2}$ and subsequent production of $\mathrm{O}_{2}{ }^{--}$and $\mathrm{H}_{2} \mathrm{O}_{2}{ }^{6}$. The complex I and III of the mETC are known as major ROS-producing sites during abiotic stress ${ }^{5}$. In plants, mETC contains an alternative respiration pathway consisting of type II $\mathrm{NAD}(\mathrm{P}) \mathrm{H}$ dehydrogenase (ND) and the alternative oxidase (AOX). The alternative respiration pathway works as a bypass of mETC and does not directly contribute to proton pumping or ATP synthesis, and excess energy is released as heat. The alternative respiration pathway is considered to be important for preventing

${ }^{1}$ Plant Genomic Network Research Team, RIKEN Center for Sustainable Resource Science (CSRS), Yokohama, 230-0045, Japan. ${ }^{2}$ Department of Advanced Bioscience, Faculty of Agriculture, Kindai University, Nara, 631-8505, Japan. ${ }^{3}$ Chemical Biology Research Group, RIKEN CSRS, Wako, Saitama, 351-0198, Japan. ${ }^{4}$ Chemical Resource Development Research Unit, RIKEN CSRS, Wako, Saitama, 351-0198, Japan. ${ }^{5}$ Gene Discovery Research Group, RIKEN CSRS, Wako, Saitama, 351-0198, Japan. ${ }^{6}$ Graduate School of Biotsudies, Kyoto University, Kyoto, 606-8502, Japan. ${ }^{7}$ School of Life Sciences, Tokyo University of Pharmacy and Life Sciences, Hachioji, Tokyo, 192-0392, Japan. ${ }^{8}$ Kihara Institute for Biological Research, Yokohama City University, Yokohama, 244-0813, Japan. ${ }^{9}$ CREST, JST, Kawaguchi, Saitama, 332-0012, Japan. ${ }^{10}$ Plant Epigenome Regulation Laboratory, RIKEN Cluster for Pioneering

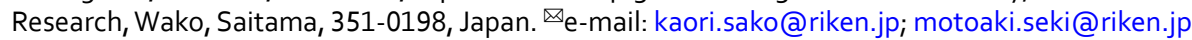


over-reduction of mETC and for mitigating ROS production ${ }^{7}$. NDs are classified into internal and external $\mathrm{NAD}(\mathrm{P}) \mathrm{H}$ dehydrogenases and reduces ubiquinone bypassing complex $\mathrm{I}^{8}$. AOX bypasses the proton-pumping complexes III and IV and acts to maintain the reduction state of the UQ pool and to lower ROS production in mitochondria 9 . In Arabidopsis, there are seven type II NDs (NDB1-4, NDA1-2 and NDC1) and five AOXs (AOX1a-d and AOX2). These alternative respiration pathway genes are induced at a gene, protein and activity level by a variety of stress conditions ${ }^{10}$, suggesting that this pathway is important for stress response.

So far, genetic approaches including conventional breeding and gene recombination have been employed as strategies to enhance stress tolerance. However, breeding is time-consuming, and genetically modified crops are unacceptable in many countries around the world. Instead, recent research suggests that the chemical priming may improve abiotic stress tolerance in plants ${ }^{11}$. We previously showed that histone deacetylase inhibitors and ethanol enhanced salinity-stress tolerance in plants including Arabidopsis, rice and cassava ${ }^{12-15}$. Those studies indicated that chemical compounds have the potential to improve tolerance in various agricultural crops. We therefore screened the RIKEN Natural Products Depository (NPDepo) chemical library to identify further compounds enhancing salinity-stress tolerance. A novel compound, FSL0260, was identified as enhancing salt-stress tolerance in plants. We demonstrated that FSL0260 works as an inhibitor of mitochondrial complex I and enhances an alternative respiration pathway, resulting in reduction of ROS accumulation and enhancement of salt-stress tolerance.

\section{Results}

FSL0260 is identified as a novel compound enhancing salt stress tolerance in A. thaliana. To identify compounds that enhance salt-stress tolerance in plants, we screened 405 compounds from the NPDepo library. Arabidopsis wild-type Columbia-0 (Col-0) seeds were grown in liquid culture medium containing each compound for 4 days, and then plants were treated with $100 \mathrm{mM} \mathrm{NaCl}$. We then observed survival rates for 4 days, and identified four compounds that increased survival rates under high salt-stress conditions. Among them, we focused on 2-[[[(4-methylphenyl)sulfonyl] oxy]methyl]-2H-1-benzopyran-3-yl]methylpyridin-1-ium 4-methylbenzenesulfonate (1:1) (FSL0260) (Fig. 1a), because it showed the strongest tolerance to salinity stress. To confirm the salinity-stress tolerance by FSL0260, wild-type plants grown in liquid culture medium for 4 days were treated with $0-40 \mu \mathrm{M}$ FSL0260 for $24 \mathrm{~h}$, with or without subsequent treatment with $100 \mathrm{mM} \mathrm{NaCl}$ for 4 days. The plants treated with FSL0260 increased their survival rate in a dose-dependent manner under salinity-stress conditions (Fig. 1b,c). We observed that the chlorophyll content of plants treated with more than $20 \mu$ M FSL0260 under salinity stress was recovered at the same level as that of plants under normal conditions (Fig. 1d), and confirmed that FSL0260 enhanced salinity-stress tolerance. However, high concentrations of FSL0260 treatment inhibited plant growth (Supplementary Fig. S1). As $20 \mu$ M FSL0260 greatly enhanced salinity-stress tolerance and minimized growth inhibition, we adopted $20 \mu \mathrm{M}$ FSL0260 for further analysis. In addition, we confirmed that FSL0260 enhanced salinity-stress tolerance not only in liquid culture but also in solid agar plates (Supplementary Fig. S2a,b).

Mitochondrial alternative respiration pathway genes are up-regulated by FSL0260 treatment under salinity stress. To elucidate the molecular mechanism of high salt-stress tolerance mediated by FSL0260, we performed global gene expression analysis with a microarray. Four-day-old plants treated with $20 \mu$ M FSL0260 or dimethyl sulfoxide (DMSO) for $24 \mathrm{~h}$ were examined. We identified 58 genes whose expression was up-regulated by $24 \mathrm{~h}$ FSL0260 treatment (Supplementary Table S1). We found that a large proportion of genes which were annotated as mitochondrial proteins were up-regulated by FSL0260 treatment (Fig. 2a). We focused particularly on mitochondrial alternative respiration pathway genes (AtAOX1a and AtNDB4), since they have been reported to detoxify ROS ${ }^{9}$. The expression profiles of AtAOX1a and AtNDB4 were confirmed by quantitative real-time PCR (qRT-PCR). The expressions of these genes were up-regulated by FSL0260 treatment (Fig. 2b). Next, we confirmed the protein levels of AOX in plants treated with FSL0260. We used non-reducing SDS-PAGE electrophoresis followed by protein gel blotting and evaluated the AOX protein level. Reduced active form AOX (about $35 \mathrm{kDa}$ ) was increased by FSL0260 treatment and by both FSL0260 and NaCl treatments (Fig. 2c,d), consistent with the transcription level of AtAOX1a under FSL0260 treatment. These results suggest that the salt tolerance conferred by FSL0260 might be due to promotion of ROS detoxification.

Mitochondrial complex I inhibitor enhances salinity-stress tolerance in A. thaliana. Previous transcriptome analysis showed that the treatment of inhibitors of the electron transport chain in mitochondria induced alternative respiration pathway genes ${ }^{16,17}$. Our transcriptome analysis showed that alternative respiration pathway genes were up-regulated by FSL0260 treatment, thus we speculated that FSL0260 works as a mitochondrial inhibitor. To verify this hypothesis, we first examined whether mitochondrial inhibitors affected salinity-stress responses. Four-day-old plants were treated with rotenone and piericidin A (which are complex I inhibitors), malonate (complex II inhibitor), antimycin A (AA) (complex III inhibitor), and KCN (complex IV inhibitor) for $24 \mathrm{~h}$, respectively. They were subsequently treated with $\mathrm{NaCl}$ for 4 days and their survival rate was checked. Plants treated with rotenone or piericidin A were able to survive in a dose-dependent manner under high salinity stress; however, malonate, AA and $\mathrm{KCN}$ were unable to rescue plants under high salt conditions (Fig. 3a,b). We confirmed that rotenone treatment, as well as the FSL0260 treatment, increased gene expressions of AtAOX1a and AtNDB4 (Supplementary Fig. S3), suggesting that the inhibition of complex I enhances salt-stress tolerance and that FSL0260 is also an inhibitor of mitochondrial complex I.

FSL0260 binds to mitochondrial complex I and inhibits its activity in plants. To investigate whether FSL0260 inhibits mitochondrial complex I activity, we isolated mitochondria from potato tubers and measured complex I activity in two ways: (1) oxygen consumption rate (OCR) was measured as an index for 
(a)<smiles>Cc1ccc(S(=O)(=O)OCC2Oc3ccccc3C=C2C[n+]2ccccc2)cc1</smiles>

(b)
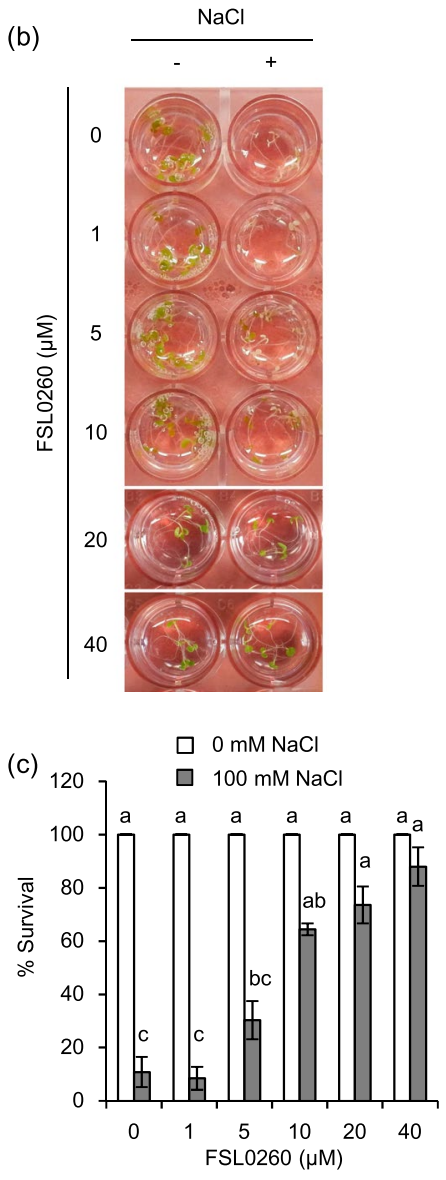

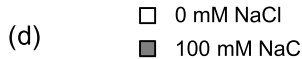

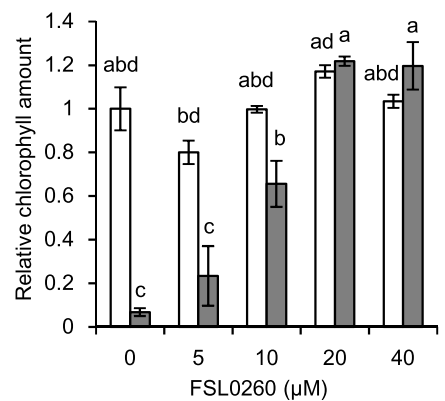

Figure 1. FSL0260 enhances high salinity stress tolerance in Arabidopsis thaliana. (a) Structure of FSL0260. (b) Morphology of seedlings treated with 0-40 $\mu$ M FSL0260, with or without a subsequent treatment of $100 \mathrm{mM}$ $\mathrm{NaCl}$ for 4 days. DMSO was used as negative control. Inside diameter of the well is $15.4 \mathrm{~mm}$. (c) Survival rate under high-salinity conditions in the presence or absence of 0-40 $\mu \mathrm{M}$ FSL0260. The survival rate of 15 plants was calculated 4 days after $\mathrm{NaCl}$ treatment. (d) Chlorophyll content in 0-40 $\mu \mathrm{M}$ FSL0260-treated plants under normal or high-salinity conditions. The chlorophyll content of the plants treated with $0 \mu \mathrm{M}$ FSL0260 was set as 1 . These experiments were conducted using three biological replicates. Error bars represent the mean \pm standard error (SE). Statistical significance was determined by ANOVA, followed by post-hoc Tukey's tests. Means that differed significantly $(\mathrm{P}<0.05)$ are indicated by different letters. 
(a)

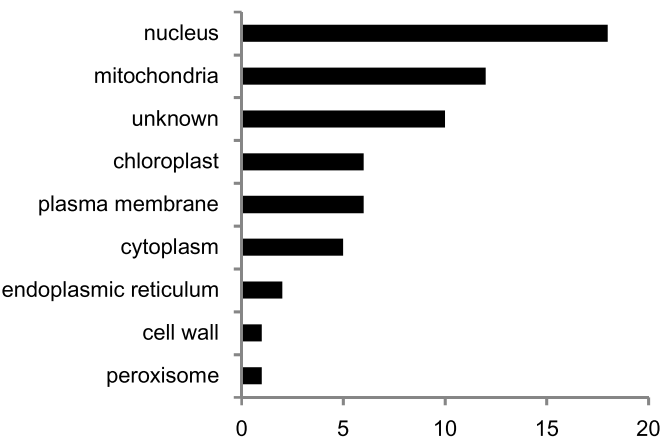

(b)
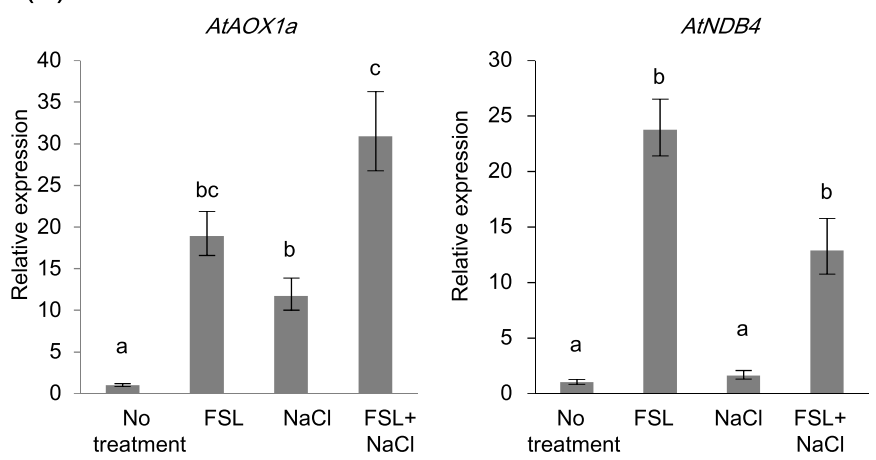

(c)
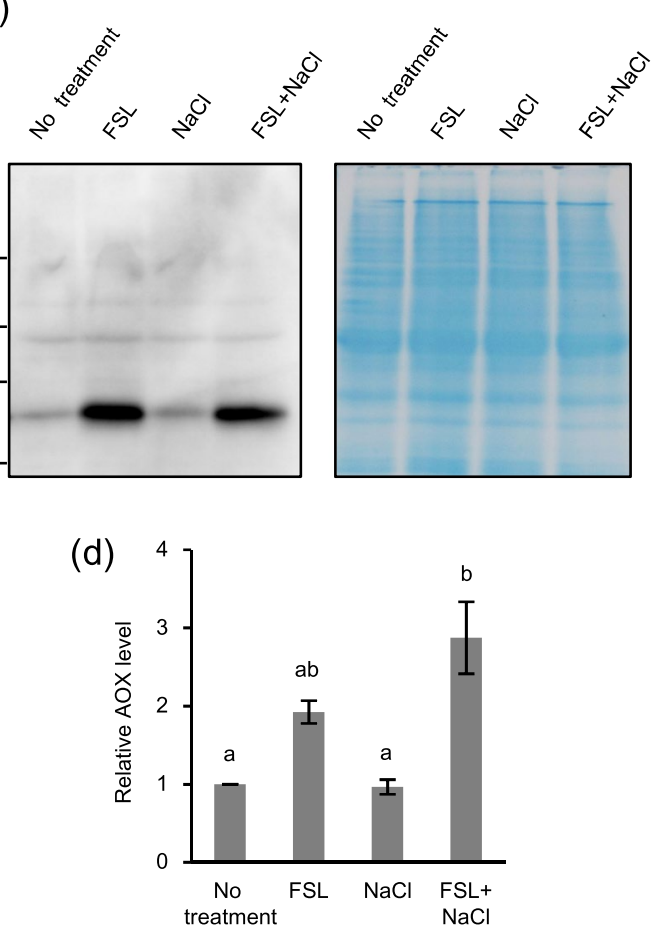

Figure 2. Expression profile of genes up-regulated by both FSL0260 treatment and salinity stress. (a) Cellular component gene ontology of up-regulated genes by FSL0260 treatment. (b) Relative expression levels of AtNDB4 and AtAOX1a genes during salinity-stress treatment for 0 and $2 \mathrm{~h}$ with or without $20 \mu \mathrm{M}$ FSL0260. Expression level of plants treated with DMSO was set as $1.18 \mathrm{~S}$ rRNA was used as an internal standard. Error bars represent the mean $\pm S E(n=3)$. Statistical significance was determined by ANOVA, followed by post-hoc Tukey's tests. Means that differed significantly $(\mathrm{P}<0.05)$ are indicated by different letters. $(\mathbf{c})$ Immunoblot of the AOX $(35 \mathrm{kDa})$ proteins (left). Coomassie blue-stained gel showing control loading (right). Total proteins were extracted from seedlings treated with 0 or $20 \mu \mathrm{M}$ FSL0260 for $24 \mathrm{~h}$ and with or without subsequent treatment of $100 \mathrm{mM} \mathrm{NaCl}$ for $6 \mathrm{~h}$. DMSO was used as a negative control. Immunoblot analysis was performed using an antiAOX1/2 antibody. (d) The signal intensity of AOX1/2. DMSO treatment was taken as 1 . Error bars represent the mean \pm SE $(n=3)$. Statistical significance was determined by ANOVA, followed by post-hoc Tukey's tests. Means that differed significantly $(\mathrm{P}<0.05)$ are indicated by different letters. 

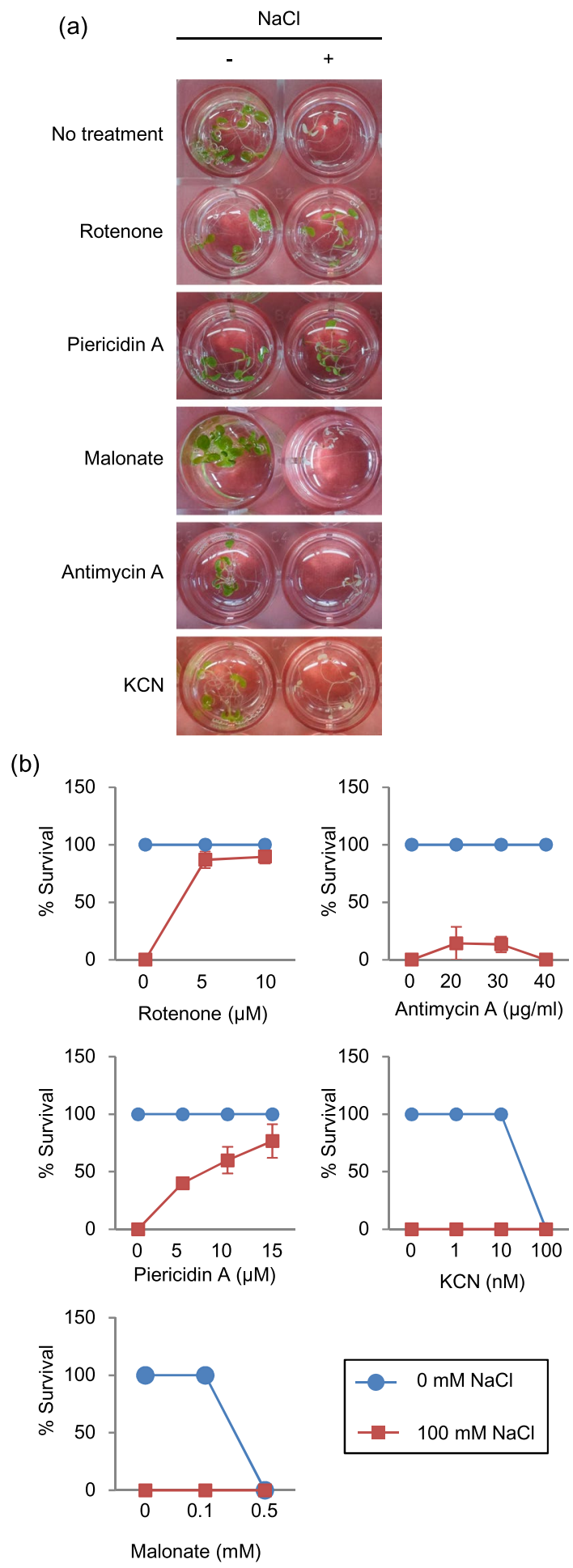

Figure 3. Inhibitors of mitochondrial complex I enhance high salinity stress tolerance. (a) Morphology of seedlings treated with $5 \mu \mathrm{M}$ rotenone, $15 \mu \mathrm{M}$ piericidin $\mathrm{A}, 0.1 \mathrm{mM}$ malonate $40 \mu \mathrm{g} / \mathrm{mL}$ antimycin A (AA) and $10 \mathrm{nM} \mathrm{KCN}$ with or without subsequent treatment with $100 \mathrm{mM} \mathrm{NaCl}$ for 4 days. DMSO was used as negative control. Inside diameter of the well is $15.4 \mathrm{~mm}$. (b) Survival rate of plants treated with various mitochondrial inhibitors under high-salinity conditions. The survival rate of 15 plants was calculated 4 days after $\mathrm{NaCl}$ treatment. Lines with circles and squares designate the survival rates of inhibitor-treated plants under normal and salt-stress growth conditions, respectively. The experiment was conducted using three biological replicates. Error bars represent the mean $\pm \mathrm{SE}$.

oxidation of deamino-NADH, which is an NADH analog and specific substrate of complex I ${ }^{18}$. FSL0260 treatment inhibits oxidation of deamino-NADH in a dose-dependent manner (Fig. 4a); and (2) we assessed oxidation of deamino-NADH by spectrometry. Results showed that FSL0260 treatment decreased oxidation of 
(a)
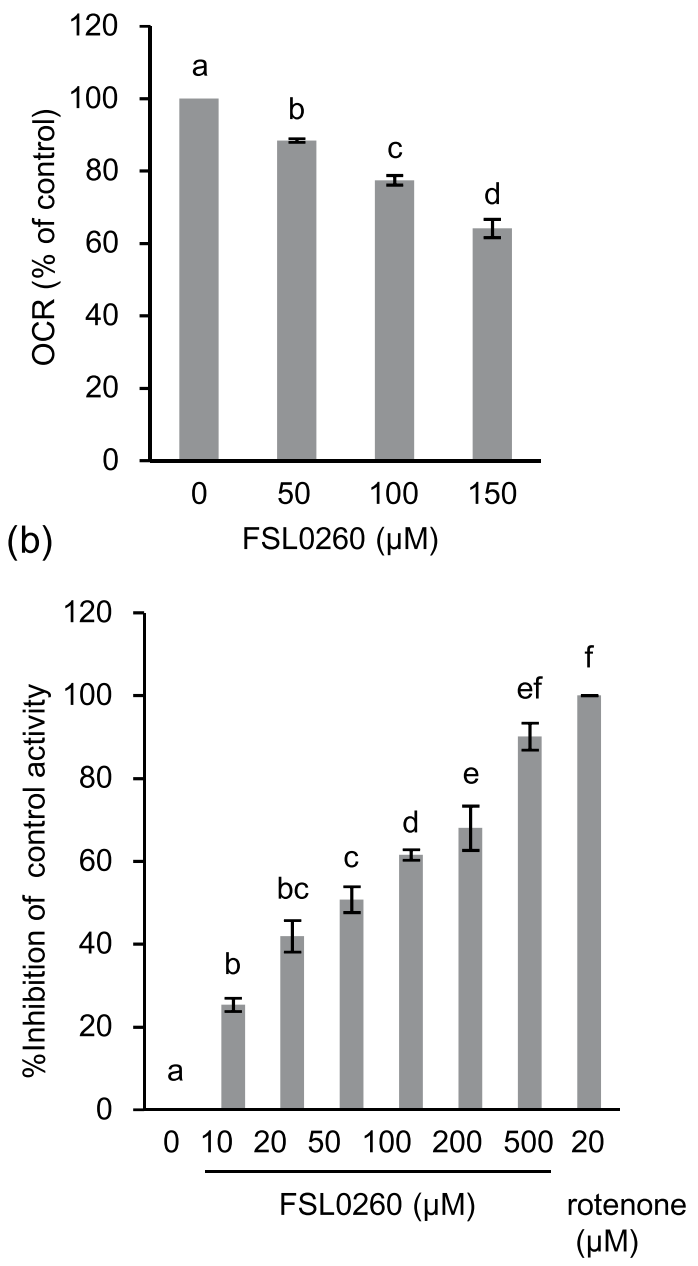

(c)

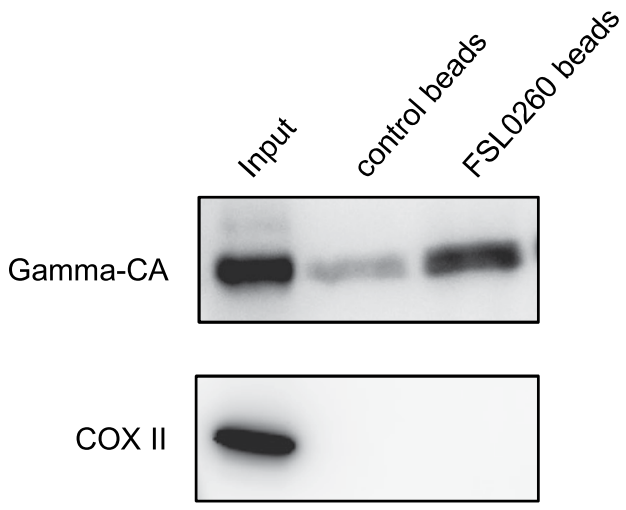

Figure 4. FSL0260 interacts with mitochondrial complex I and inhibits its activity. (a) Inhibition of complex I by FSL0260. Oxygen consumption rate (OCR) of isolated mitochondria from potato tuber was monitored with deamino-NADH in the absence or presence of FSL0260. (b) Inhibition of deamino-NADH oxidation by FSL0260. Deamino-NADH oxidation was measured by spectrometry using sonicated mitochondria isolated from the potato tuber. The experiment was conducted using three biological replicates. Error bars represent the mean $\pm \mathrm{SE}(\mathrm{n}=3)$. (c) Pull-down assay of FLS0260. Sonicated potato mitochondria were incubated with control or FSL0260 beads. Immunoblot assay was performed using an anti-gamma CA antibody and an antiCOX II antibody.

deamino-NADH in a concentration-dependent manner (Fig. 4b), suggesting that FSL0260 has inhibition activity in respect of mitochondrial complex I $(\mathrm{IC} 50=45.1 \mu \mathrm{M})$. Furthermore, we measured OCR for assessing activity of complex IV and activity of complex II by spectrometry. These results confirmed that FSL0260 did not inhibit 


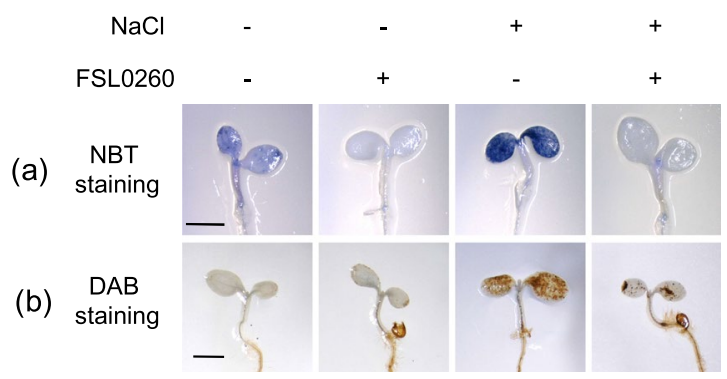

Figure 5. ROS accumulation is reduced by FSL0260 treatment. NBT and DAB staining were used to assess the accumulation of $\mathrm{O}_{2} \bullet-$ and $\mathrm{H}_{2} \mathrm{O}_{2}$, respectively. Arabidopsis thaliana plants were treated with $\mathrm{NaCl}$ for $6 \mathrm{~h}$ in the presence or absence of $20 \mu \mathrm{M}$ FSL0260. Bar $=1 \mathrm{~mm}$. Each treatment was analyzed using 10 plants. Three biological repeats were performed.

complex II and IV (Supplementary Fig. S4a,b). We also assessed whether FSL0260 worked in mammalian mitochondria, and found that it did not inhibit mitochondria isolated from the bovine heart (Supplementary Fig. S5), suggesting that FSL0260 preferably inhibits plant mitochondria.

To confirm whether FSL0260 binds to mitochondrial complex I, a pull-down assay was performed using FSL0260-fixed agarose beads. Gamma-CA, a subunit of mitochondrial complex I, was significantly pulled down with FSL0260-bound beads. Control beads bound only slightly with gamma-CA (Fig. 4c). COX II, a subunit of mitochondrial complex IV, was not pulled down with both control beads and FSL0260-bound beads (Fig. 4c), indicating the physical interaction of FSL0260 and mitochondrial complex I. These results revealed that FSL0260 is a novel inhibitor of mitochondrial complex I in plants.

FSL0260 enhances the detoxification of ROS under high salinity-stress conditions. The accumulation of $\mathrm{O}_{2}{ }^{--}$and $\mathrm{H}_{2} \mathrm{O}_{2}$, which are the two main ROS components induced by salt stress, normally results in oxidative damage. We investigated the accumulation of $\mathrm{O}_{2}{ }^{--}$and $\mathrm{H}_{2} \mathrm{O}_{2}$ in FSL0260-treated plants under salt-stress conditions using NBT to detect $\mathrm{O}_{2}{ }^{--}$and DAB staining for monitoring $\mathrm{H}_{2} \mathrm{O}_{2}$. The cotyledons of NaCl-treated plants were significantly stained by NBT and DAB, indicating that $\mathrm{O}_{2}{ }^{\bullet-}$ or $\mathrm{H}_{2} \mathrm{O}_{2}$ were highly accumulated under salinity-stress conditions. NBT staining showed that FSL0260 treatment led to a greatly reduced accumulation of $\mathrm{O}_{2}{ }^{--}$in plants (Fig. 5a). DAB staining also showed decreased accumulation of $\mathrm{H}_{2} \mathrm{O}_{2}$ by FSL0260 treatment (Fig. 5b). Some chemical compounds - such as ascorbic acid - have radical scavenging activity, thus we confirmed that FSL0260 itself did not show antioxidant activity (Supplementary Fig. S6a,b). FSL0260 treatment induced AOX, thus we investigated ROS level of plants treated with both FSL0260 and salicylhydroxamic acid (SHAM) which is an inhibitor of AOX. The reduction of $\mathrm{O}_{2}{ }^{--}$by FSL0260 under salt stress was repressed by SHAM (Supplementary Fig. S7). These data suggested that up-regulation of alternative respiration pathway by FSL0260 detoxifies ROS and enhances salinity-stress tolerance in A. thaliana.

FSL0260 treatment enhances high-salinity tolerance by decreasing the accumulation of ROS in rice. To confirm whether FSL0260 enhances the tolerance of monocots to salt stress condition, 8-day-old rice seedlings were treated with FSL0260 for 2 days, and then plants were treated with $250 \mathrm{mM} \mathrm{NaCl}$ for 7 days. Under normal condition, no significant morphological differences were observed between control plants and FSL0260 treated plants (Fig. 6a). On the other hand, FSL0260 treated plants showed increased shoot growth compared to non-treated plants under salt stress condition (Fig. 6b,c), suggesting that FSL0260 enhances salinity stress tolerance in rice as well as $A$. thaliana.

FSL0260 treatments enhanced ROS detoxification and improved the salt stress tolerance of $A$. thaliana plants. We verified that salinity stress tolerance in rice is also due to the detoxification of ROS by NBT and DAB staining. Rice leaves were more extensively stained by NBT and DAB under salinity stress condition than under control condition (Fig. 6d). However, the intensity of the NBT and DAB staining decreased in FSL0260 treated plants under salt stress condition, suggesting that FSL0260 inhibited ROS accumulation (Fig. 6d), implying that FSL0260 increases salinity tolerance in rice by inhibiting ROS accumulation, similar to its effects in A. thaliana.

\section{Discussion}

In this study, we identified a novel compound FSL0260 enhancing salinity-stress tolerance from the chemical screening. The results suggest that FSL0260 has inhibitory activity of mitochondrial complex I and this inhibition induces an alternative respiration pathway including AtAOX1a and AtNDB4. Up-regulation of an alternative respiration pathway by FSL0260 treatment is associated with reduction in ROS accumulation, leading to enhanced salinity-stress tolerance in plants.

Our results are consistent with a previous report showing that Arabidopsis plants overexpressing AtAOX1a genes exhibited increased salt-stress tolerance following lower ROS levels under salinity stress ${ }^{19}$. The RNAi line of AtNDB4 showed reduction in ROS accumulation and salt-stress tolerance caused by enhancing AtNDB2 and $A t A O X 1 a^{20}$, and plant overexpressing both AtNDB2 and AtAOX1a showed increased NADH oxidation rates and enhanced tolerance to drought and high-light stress ${ }^{21}$, suggesting that there is functional link between AtNDB and AtAOX and total amount of alternative respiration pathway-related genes affects ROS detoxifying activity. 
(a)

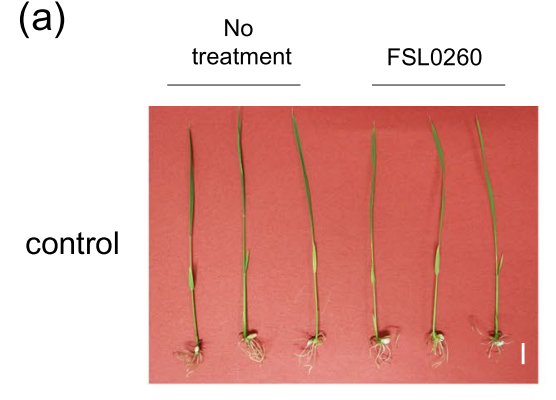

(b)

No

$$
\text { treatment FSL0260 }
$$

$250 \mathrm{mM}$

$\mathrm{NaCl}$

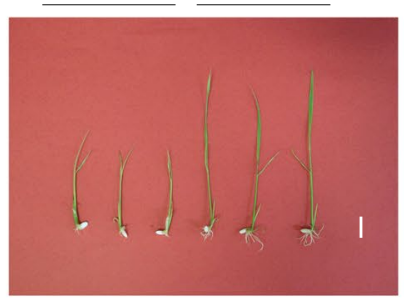

(c)

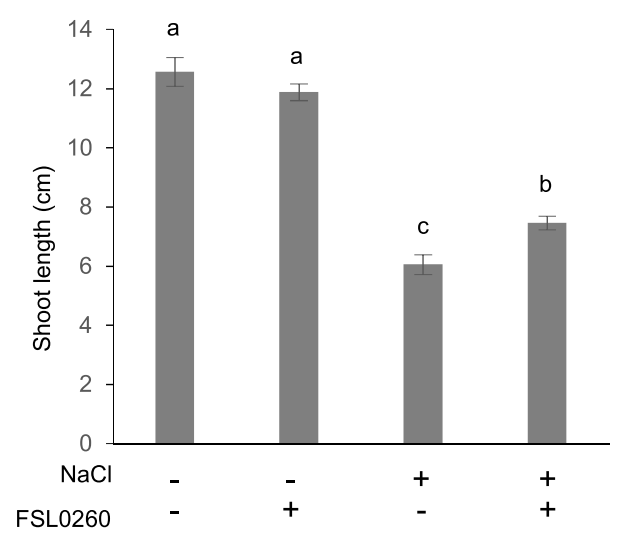

(d)

$\mathrm{NaCl}$
FSL0260

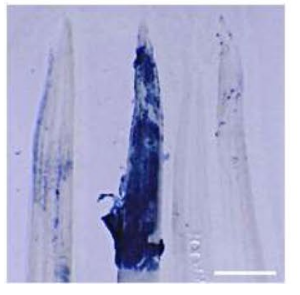

NBT staining

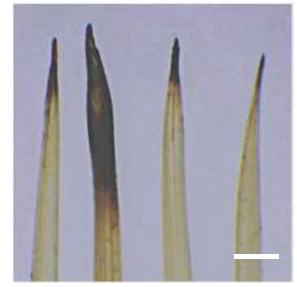

DAB staining

Figure 6. FSL0260 enhances high salinity stress tolerance in Oryza sativa. Morphology of rice seedlings without $\mathrm{NaCl}(\mathbf{a})$, and treated with $250 \mathrm{mM} \mathrm{NaCl}$ for 7 days in the presence or absence of $20 \mu \mathrm{M} \mathrm{FSL0260} \mathrm{(b).}$ DMSO was used as negative control. $\mathrm{Bar}=1 \mathrm{~cm}$. (c) Shoot length of rice seedlings under normal condition and salt stress. (d) NBT and DAB staining were used to assess the accumulation of $\mathrm{O}_{2}{ }^{\bullet-}$ and $\mathrm{H}_{2} \mathrm{O}_{2}$, respectively. 7 -day-old rice were treated with $\mathrm{NaCl}$ for $24 \mathrm{~h}$ in the presence or absence of $20 \mu \mathrm{M}$ FSL0260. Bar $=1 \mathrm{~mm}$. Three biological repeats were performed.

Thus, upregulation of both AtNDB4 and AtAOX1a by FSL0260 treatment probably contributes to decrease ROS accumulation and enhances salt stress tolerance.

Our microarray data indicated that the expression of some mitochondrial stress responsive genes was upregulated by FSL0260 treatment. Previous report showed that the expression of AT12CYS-2 was induced by inactivation of mitochondrial complex I ${ }^{22}$. AtNAC013 and AtNAC044, NAC family transcription factors, have been demonstrated to play crucial roles in mitochondrial retrograde signaling ${ }^{23,24}$. AtNAC013 induces transcription of 
AtAOX1a and AT12CYS-2 under mitochondrial stress, and Arabidopsis plants overexpressing AtNAC013 genes exhibited increased oxidative-stress tolerance following lower ROS levels under salinity stress ${ }^{24}$. These data provided support the observation that FSL0260 inhibits mitochondrial complex I activity and induces the alternative respiration pathway.

Mitochondrial complex I in Arabidopsis is composed of at least 49 subunits, 40 of which represent homologs of bovine complex I and 9 of which are unique to plants ${ }^{25,26}$. In rice, 33 subunits were classified as the component of complex I ${ }^{27,28}$ and they showed the high conservation with that in Arabidopsis thaliana ${ }^{29}$. Some deficient mutants of complex I have been reported to improve abiotic stress tolerance in plants. CMSII mutant that has deletion of the NAD7 in tobacco (Nicotiana sylvestris) was found to be more resistant to ozone stress ${ }^{30}$. And bir6 mutant which is abnormal splicing of NAD7 subunit, enhanced salt and osmotic stress tolerance in Arabidopsis ${ }^{31}$. The $n d u f s 4$ mutant (the T-DNA insertion mutant of NDUFS4-encoded $18 \mathrm{kDa}$ Fe-S protein of complex I in Arabidopsis) showed enhanced tolerance to cold, mild salt and osmotic stress ${ }^{32}$. Although ROS levels under stress conditions have not been mentioned in these mutants, transcription of the alternative respiration pathway was up-regulated in the CMSII, $n d u f_{s} 4$ and bir6 mutant ${ }^{33}$. In addition, a mutant of OsNDUFA9 encoding a mitochondrial complex I subunit in rice was embryo lethal but OsAOX1a and OsAOX1b were upregulated in the mutant ${ }^{34}$. These observations support our conclusion that inhibition of mitochondrial complex I by FSL0260 enhances an alternative respiration pathway and detoxifies ROS under salinity stress.

Previous reports described that AA treatment induced the expression of AtAOX1a and AtNDB422,35. However, our result showed that AA treatment did not rescue plants from salinity stress. To investigate cause of this discrepancy, we analyzed ROS accumulation in plants treated with AA. The accumulations of $\mathrm{O}_{2}{ }^{--}$were not significantly different between plants treated with and without AA under salt stress condition (Supplementary Fig. 8), indicating that AA could not repress ROS production even though alternative respiration pathway was activated. We speculated that AOX activation could not fully compensate the inhibition of complex III by AA, then ROS was accumulated in plants treated with AA. The ppr40-1 knockout mutant which impaired electron transport through complex III, enhanced ROS accumulation and showed the increased sensitivity to salt stress, although gene expression of AOX was upregulated in this mutant ${ }^{36}$, supporting our speculation.

In addition to FSL0260, rotenone and piericidin A also enhanced salinity-stress tolerance in plants. Compared with rotenone, the results suggested that FSL0260 preferentially inhibits plant mitochondrial complex I due to its binding site. Rotenone has been reported to bind to the quinone-binding pocket formed by the PSST, ND1 and the $49-\mathrm{kDa}$ subunit, which are conserved in both mammals and plants ${ }^{37,38}$. It is possible that FSL0260 binds to plant-specific subunits of mitochondrial complex I, resulting in its preference. Fenpyroximate, a mitochondrial complex I inhibitor, displays a selective toxicity to mites and was marketed as an acaricide in the $1990 \mathrm{~s}^{39}$. This selectivity was caused by rapid hydrolysis in mammals compared with mites ${ }^{40,41}$, suggesting that selectivity in species has advantages in agricultural applications. Although a toxicity test using experimental animals is required to confirm the selectivity of FSL0260, we may expect that FSL0260 could be used as a vitalizing agent in agriculture to enhance salinity-stress tolerance without toxicity to humans.

\section{Methods}

Plant materials and growth conditions. A. thaliana (ecotype Columbia-0) seeds were sterilized and sown in half-strength Murashige and Skoog (MS) liquid medium supplemented with $1 \%$ sucrose and $0.1 \%$ agar. The plants were grown under previously described conditions ${ }^{15}$. Four-day-old plants were treated with compounds for $24 \mathrm{~h}$, with or without subsequent treatment with $100 \mathrm{mM} \mathrm{NaCl}$ (WAKO, Japan). The $\mathrm{NaCl}$ solution was added into the medium containing both compound and plants. The survival rate of 20 plants was calculated 4 days after the $\mathrm{NaCl}$ treatment. The experiment was conducted using three biological replicates.

For rice experiments, Oryza sativa L. cv. Nipponbare seeds were germinated in water at $28^{\circ} \mathrm{C}$ for 3 days, and then transferred to $50 \mathrm{ml}$ tube containing $1 / 2 \mathrm{MS}$ liquid medium and growth (16-h-light/8-h-dark cycle at $22^{\circ} \mathrm{C}$ ). For salt stress test, 8-day-old plants were treated with FSL0260 and incubated for 2 days with or without subsequent treatment with $250 \mathrm{mM} \mathrm{NaCl}$ for 7 days. Seedling length was measured using ImageJ software (https:// imagej.nih.gov/ij/index.html). The data was analyzed from 12 plants for each treatment. The experiment was conducted using two independent biological replicates.

Chemical materials. The chemical library $\left(10 \mathrm{mg} \mathrm{mL}^{-1}\right.$ of each compound in dimethylsulfoxide (DMSO, $5 \mu \mathrm{L})$ ) was obtained from the RIKEN NPDepo (http://www.cbrg.riken.jp/npedia/?LANG=en). The synthesis of FSL0260 is described in Supplemental methods. Rotenone and Antimycin A (AA) were purchased from Sigma-Aldrich (St. Louis, USA). KCN and Malonate were purchased from WAKO (Osaka, Japan). Piericidin A was purchased from BioAustralis (Smithfield, Australia). Salicylhydroxamic acid was purchased from Tokyo Chemical Industry Co., Ltd. (Tokyo, Japan).

Chemical library screening. We sowed 5-6 Col-0 seeds in each well containing $500 \mu \mathrm{L}$ of half-strength MS liquid medium containing $10 \mu \mathrm{g} \mathrm{mL}^{-1}$ chemical in a 48 -well plate. Seedlings were grown for 5 days and $100 \mathrm{mM}$ $\mathrm{NaCl}$ was added. The survival rates were checked 4 days after the $\mathrm{NaCl}$ treatment.

Measurement of chlorophyll content. Four-day-old A. thaliana plants were treated with 0-40 $\mu \mathrm{M}$ FSL0260 for $24 \mathrm{~h}$ and then exposed to $100 \mathrm{mM} \mathrm{NaCl}$ for $72 \mathrm{~h}$. We then measured the chlorophyll content of $30-50 \mathrm{mg}$ of seedlings for each treatment as previously described ${ }^{15}$. The experiment was conducted with three biological replicates. Statistical significance was determined by ANOVA, followed by post-hoc Tukey's tests. Means that differed significantly $(\mathrm{P}<0.05)$ are indicated by different letters. 
RNA extraction. Total RNA was extracted from 5-day-old A. thaliana seedlings treated with $20 \mu \mathrm{M}$ FSL0260 for $24 \mathrm{~h}$, with or without subsequent treatment with $100 \mathrm{mM} \mathrm{NaCl}$ for $2 \mathrm{~h}$. DMSO was used as a negative control. For qRT-PCR, total RNA was extracted from 5-10 plants using the Plant RNA reagent (Thermo Fisher Scientific, Waltham, USA) as previously described ${ }^{42}$. For microarray analysis, total RNA was extracted from 20 plants with an RNeasy Plant Mini Kit (Qiagen, Hilden Germany) according to the manufacturer's instructions. The quality of the extracted total RNA was evaluated using a Bioanalyzer system (Agilent, Santa Clara, USA). The experiment was conducted using three biological replicates.

Microarray analysis. A microarray analysis was completed as previously described ${ }^{42}$. The microarray data were deposited in the GEO database (GEO ID: GSE128820). Each treatment was analyzed using three biological replicates, and a total of ten plants was sampled for each treatment and/or repeat. Genes with an expression log2 ratio $\geq 1$ [t test analysis, Benjamini-Hochberg correction $(\mathrm{FDR})<0.05$ ] were identified as up-regulated genes. Gene Ontology assignments for Arabidopsis genes were obtained from TAIR (www.arabidopsis.org).

Quantitative real-time PCR analysis. First-strand cDNA synthesis was performed with a PrimeScript ${ }^{\mathrm{TM}}$ RT reagent Kit (Takara, Kusatsu Japan) for quantitative real-time polymerase chain reaction (qRT-PCR) analysis. The qRT-PCR was conducted as previously described ${ }^{15}$ and $18 \mathrm{~S}$ rRNA was used as a reference gene. The experiment was conducted using three biological replicates, and a total of ten plants was sampled for each treatment and/or repeat. The qRT-PCR primer sequences are shown in Table S2.

Protein extraction and immunoblot analysis. Seedlings were pulverized in liquid nitrogen with a multi beads shocker (Yasui Kikai, Osaka, Japan), and an SDS sample buffer (without $\beta$-mercaptoethanol) was added. After centrifugation at $15,000 \mathrm{rpm}$ for $5 \mathrm{~min}$ at $4{ }^{\circ} \mathrm{C}$, the supernatants were transferred into clean tubes. Total proteins were separated onto $12.5 \%(\mathrm{w} / \mathrm{v})$ acrylamide gels by non-reducing SDS-PAGE. Immunoblotting was performed as described previously ${ }^{43}$ using anti-AOX1/2 (AS04 054), anti-Gamma CA (AS17 4114) and anti-COX II (AS04 053A) (Agrisera, Vännäs, Sweden).

Isolation of mitochondria. Potato tubers (Solanum tuberosum L.) were purchased from local markets. Isolation of intact mitochondria from potato tubers was performed according to Yoshida et al. ${ }^{44}$.

Measurement of oxygen uptake rate by isolated mitochondria. Oxygen uptake rates were measured polarographically with a Clark-type oxygen electrode (Rank Brothers, Cambridge, UK) at $25^{\circ} \mathrm{C}$ in $1 \mathrm{~mL}$ of air-saturated medium containing $0.3 \mathrm{M}$ sucrose, $10 \mathrm{mM} \mathrm{KH}_{2} \mathrm{PO}_{4}, 10 \mathrm{mM}$ TES and $10 \mathrm{mM} \mathrm{MgCl}_{2}(\mathrm{pH} 6.8$ ). NADH:ubiquinone oxidoreductase activity was measured for $40 \mu \mathrm{g}$ of mitochondria which were freeze-thawed three times in the presence of $1 \mathrm{mM}$ deamino-NADH (Sigma-Aldrich, St. Louis, USA). Percentage FSL0260 resistance was calculated using the formula in Eq. (1):

$$
\% \text { FSL0260 resistance }=\{[\text { Rate }+ \text { FSL0260] } /[\text { Rate }- \text { FSL0260] }\} \times 100 \%
$$

where Rate is the rate of oxygen consumption (nmol oxygen $\mathrm{min}^{-1} \mathrm{mg}^{-1}$ of protein).

Measurement of enzymatic activity. Complex I activity was measured by a modified version of a published method ${ }^{45,46}$. Mitochondria were suspended in $0.35 \mathrm{M}$ mannitol, $0.25 \mathrm{M}$ sucrose and $25 \mathrm{mM}$ TES (pH 7.8). The suspension was sonicated for $6 \times 5 \mathrm{~s}$ (maximum output), and the debris was removed by centrifugation for $10 \mathrm{~min}$ at $16,000 \mathrm{~g}$. The supernatant was used for the experiments. The rate of deamino-NADH oxidation by $0.7 \mu \mathrm{g}$ sonicated mitochondria was measured spectrophotometrically at $340 \mathrm{~nm}$ in a medium containing $50 \mathrm{mM}$ potassium buffer (pH7.5), $1 \mathrm{mM}$ EDTA, $2 \mathrm{mM} \mathrm{KCN}$ and $25 \mu \mathrm{M}$ ubiquinone at $30^{\circ} \mathrm{C}$. The reaction was started by adding $0.2 \mathrm{mM}$ deamino-NADH.

Pull-down assay. FSL0260 beads were prepared as previously described ${ }^{47}$. We incubated $150 \mu$ g sonicated potato mitochondria with FSL0260 beads in $1 \mathrm{~mL}$ binding buffer containing $50 \mathrm{mM}$ Tris $\mathrm{HCl}(\mathrm{pH} 7.5), 150 \mathrm{mM}$ $\mathrm{NaCl}, 0.5 \%$ Tween 20, $0.1 \mathrm{mM}$ EDTA and Protease Inhibitor Cocktail for plant cells (Sigma-Aldrich St. Louis, USA) for $3 \mathrm{~h}$ at $4{ }^{\circ} \mathrm{C}$. The beads were then washed three times in $1 \mathrm{~mL}$ binding buffer and eluted with an SDS sample buffer. The eluted protein was then subjected to SDS-PAGE.

Staining to detect superoxide anion and hydrogen peroxide. Five-day-old $A$. thaliana plants treated with $20 \mu \mathrm{M}$ FSL0260 for $24 \mathrm{~h}$, with or without a subsequent treatment with $100 \mathrm{mM} \mathrm{NaCl}$ for $6 \mathrm{~h}$ and $5 \mathrm{~h}$, were stained for NBT and DAB. For the rice experiments, 8-day-old O. sativa L. cv. Nipponbare plants treated with $20 \mu \mathrm{M}$ FSL0260 for $24 \mathrm{~h}$, with or without a subsequent treatment with $200 \mathrm{mM} \mathrm{NaCl}$ for $24 \mathrm{~h}$, were stained for NBT and DAB. NBT and DAB staining was conducted as previously described ${ }^{13}$.

Received: 10 January 2020; Accepted: 6 May 2020;

Published online: 26 May 2020

\section{References}

1. Munns, R. \& Tester, M. Mechanisms of salinity tolerance. Annu Rev Plant Biol 59, 651-681, https://doi.org/10.1146/annurev. arplant.59.032607.092911 (2008).

2. Deinlein, U. et al. Plant salt-tolerance mechanisms. Trends Plant Sci 19, 371-379, https://doi.org/10.1016/j.tplants.2014.02.001 (2014).

3. Hossain, M. S. \& Dietz, K. J. Tuning of Redox Regulatory Mechanisms, Reactive Oxygen Species and Redox Homeostasis under Salinity Stress. Front Plant Sci 7, 548, https://doi.org/10.3389/fpls.2016.00548 (2016). 
4. Moller, I. M., Jensen, P. E. \& Hansson, A. Oxidative modifications to cellular components in plants. Annu Rev Plant Biol 58, 459-481, https://doi.org/10.1146/annurev.arplant.58.032806.103946 (2007).

5. Miller, G., Suzuki, N., Ciftci-Yilmaz, S. \& Mittler, R. Reactive oxygen species homeostasis and signalling during drought and salinity stresses. Plant Cell Environ 33, 453-467, https://doi.org/10.1111/j.1365-3040.2009.02041.x (2010).

6. Liberatore, K. L., Dukowic-Schulze, S., Miller, M. E., Chen, C. \& Kianian, S. F. The role of mitochondria in plant development and stress tolerance. Free Radic Biol Med 100, 238-256, https://doi.org/10.1016/j.freeradbiomed.2016.03.033 (2016).

7. Wanniarachchi, V. R. et al. Alternative Respiratory Pathway Component Genes (AOX and ND) in Rice and Barley and Their Response to Stress. Int J Mol Sci 19, https://doi.org/10.3390/ijms19030915 (2018).

8. Rasmusson, A. G., Soole, K. L. \& Elthon, T. E. Alternative NAD(P)H dehydrogenases of plant mitochondria. Annu Rev Plant Biol 55, 23-39, https://doi.org/10.1146/annurev.arplant.55.031903.141720 (2004).

9. Vanlerberghe, G. C. Alternative oxidase: a mitochondrial respiratory pathway to maintain metabolic and signaling homeostasis during abiotic and biotic stress in plants. Int J Mol Sci 14, 6805-6847, https://doi.org/10.3390/ijms14046805 (2013).

10. Clifton, R., Millar, A. H. \& Whelan, J. Alternative oxidases in Arabidopsis: a comparative analysis of differential expression in the gene family provides new insights into function of non-phosphorylating bypasses. Biochim Biophys Acta 1757, 730-741, https://doi. org/10.1016/j.bbabio.2006.03.009 (2006).

11. Savvides, A., Ali, S., Tester, M. \& Fotopoulos, V. Chemical Priming of Plants Against Multiple Abiotic Stresses: Mission Possible? Trends Plant Sci 21, 329-340, https://doi.org/10.1016/j.tplants.2015.11.003 (2016).

12. Ueda, M. et al. The distinct roles of class I and II RPD3-like histone deacetylases in salinity stress response. Plant Physiol, https://doi. org/10.1104/pp.17.01332 (2017).

13. Nguyen, H. M. et al. Ethanol Enhances High-Salinity Stress Tolerance by Detoxifying Reactive Oxygen Species in Arabidopsis thaliana and Rice. Frontiers in Plant Science 8, https://doi.org/10.3389/fpls.2017.01001 (2017).

14. Patanun, O. et al. The Histone Deacetylase Inhibitor Suberoylanilide Hydroxamic Acid Alleviates Salinity Stress in Cassava. Front Plant Sci 7, 2039, https://doi.org/10.3389/fpls.2016.02039 (2016).

15. Sako, K. et al. Ky-2, a Histone Deacetylase Inhibitor, Enhances High-Salinity Stress Tolerance in Arabidopsis thaliana. Plant Cell Physiol 57, 776-783, https://doi.org/10.1093/pcp/pcv199 (2016).

16. Garmier, M. et al. In Plant Physiol Vol. 148 1324-1341 (2008).

17. Umbach, A. L. et al. Comparison of intact Arabidopsis thaliana leaf transcript profiles during treatment with inhibitors of mitochondrial electron transport and TCA cycle. Plos One 7, e44339, https://doi.org/10.1371/journal.pone.0044339 (2012).

18. Huang, S., Lee, C. P. \& Millar, A. H. Activity Assay for Plant Mitochondrial Enzymes|SpringerLink. Vol. 1305 (Humana Press, 2015),

19. Smith, C. A., Melino, V. J., Sweetman, C. \& Soole, K. L. Manipulation of alternative oxidase can influence salt tolerance in Arabidopsis thaliana. Physiol Plant 137, 459-472, https://doi.org/10.1111/j.1399-3054.2009.01305.x (2009).

20. Smith, C. et al. Alterations in the mitochondrial alternative NAD(P)H Dehydrogenase NDB4 lead to changes in mitochondrial electron transport chain composition, plant growth and response to oxidative stress. Plant Cell Physiol 52, 1222-1237, https://doi. org $/ 10.1093 / \mathrm{pcp} / \mathrm{pcr} 073$ (2011).

21. Sweetman, C. et al. AtNDB2 Is the Main External NADH Dehydrogenase in Mitochondria and Is Important for Tolerance to Environmental Stress. Plant Physiol 181, 774-788, https://doi.org/10.1104/pp.19.00877 (2019).

22. Wang, Y. et al. Inactivation of Mitochondrial Complex I Induces the Expression of a Twin Cysteine Protein that Targets and Affects Cytosolic, Chloroplastidic and Mitochondrial Function. Molecular Plant 9, 696-710, https://doi.org/10.1016/j.molp.2016.01.009 (2016).

23. Leister, D. et al. Identification of Target Genes and Transcription Factors Implicated in Translation-Dependent Retrograde Signaling in Arabidopsis. Molecular Plant 7, 1228-1247, https://doi.org/10.1093/mp/ssu066 (2014).

24. De Clercq, I. et al. The membrane-bound NAC transcription factor ANAC013 functions in mitochondrial retrograde regulation of the oxidative stress response in Arabidopsis. Plant Cell 25, 3472-3490, https://doi.org/10.1105/tpc.113.117168 (2013).

25. Klodmann, J., Sunderhaus, S., Nimtz, M., Jansch, L. \& Braun, H. P. Internal architecture of mitochondrial complex I from Arabidopsis thaliana. Plant Cell 22, 797-810, https://doi.org/10.1105/tpc.109.073726 (2010).

26. Peters, K., Belt, K. \& Braun, H. P. 3D Gel Map of Arabidopsis Complex I. Front Plant Sci 4, 153, https://doi.org/10.3389/ fpls.2013.00153 (2013).

27. Chen, B. et al. Composition of Mitochondrial Complex I during the Critical Node of Seed Aging in Oryza sativa. J Plant Physiol 236, 7-14, https://doi.org/10.1016/j.jplph.2019.02.008 (2019).

28. Heazlewood, J. L., Howell, K. A. \& Millar, A. H. Mitochondrial complex I from Arabidopsis and rice: orthologs of mammalian and fungal components coupled with plant-specific subunits. Biochim Biophys Acta 1604, 159-169, https://doi.org/10.1016/s00052728(03)00045-8 (2003).

29. Huang, S. et al. Experimental analysis of the rice mitochondrial proteome, its biogenesis, and heterogeneity. Plant Physiol 149, 719-734, https://doi.org/10.1104/pp.108.131300 (2009).

30. Dutilleul, C. et al. Leaf mitochondria modulate whole cell redox homeostasis, set antioxidant capacity, and determine stress resistance through altered signaling and diurnal regulation. Plant Cell 15, 1212-1226, https://doi.org/10.1105/tpc.009464 (2003).

31. Koprivova, A. et al. Identification of a pentatricopeptide repeat protein implicated in splicing of intron 1 of mitochondrial nad7 transcripts. J Biol Chem 285, 32192-32199, https://doi.org/10.1074/jbc.M110.147603 (2010).

32. Meyer, E. H. et al. Remodeled respiration in ndufs 4 with low phosphorylation efficiency suppresses Arabidopsis germination and growth and alters control of metabolism at night. Plant Physiol 151, 603-619, https://doi.org/10.1104/pp.109.141770 (2009).

33. Juszczuk, I. M., Szal, B. \& Rychter, A. M. Oxidation-reduction and reactive oxygen species homeostasis in mutant plants with respiratory chain complex I dysfunction. Plant Cell Environ 35, 296-307, https://doi.org/10.1111/j.1365-3040.2011.02314.x (2012).

34. Hu, T. et al. OsNDUFA9 encoding a mitochondrial complex I subunit is essential for embryo development and starch synthesis in rice. Plant Cell Rep 37, 1667-1679, https://doi.org/10.1007/s00299-018-2338-x (2018).

35. Ng, S. et al. A membrane-bound NAC transcription factor, ANAC017, mediates mitochondrial retrograde signaling in Arabidopsis. Plant Cell 25, 3450-3471, https://doi.org/10.1105/tpc.113.113985 (2013).

36. Zsigmond, L. et al. Arabidopsis PPR40 connects abiotic stress responses to mitochondrial electron transport. Plant Physiol 146, 1721-1737, https://doi.org/10.1104/pp.107.111260 (2008).

37. Degli Esposti, M. Inhibitors of NADH-ubiquinone reductase: an overview. Biochim Biophys Acta 1364, 222-235, https://doi. org/10.1016/S0005-2728(98)00029-2 (1998)

38. Subrahmanian, N., Remacle, C. \& Hamel, P. P. Plant mitochondrial Complex I composition and assembly: A review. Biochim Biophys Acta 1857, 1001-1014, https://doi.org/10.1016/j.bbabio.2016.01.009 (2016).

39. Murai, M. \& Miyoshi, H. Current topics on inhibitors of respiratory complex I. Biochim Biophys Acta 1857, 884-891, https://doi. org/10.1016/j.bbabio.2015.11.009 (2016).

40. Nishizawa., H. et al. Metabolism of Fenpyroximate in Rats. Journal of Pesticide Science 18, 59-66, https://doi.org/10.1584/ jpestics.18.59 (1993).

41. Motoba, K., Suzuki, T. \& Uchida, M. Effect of a new acaricide, fenpyroximate, on energy metabolism and mitochondrial morphology in adult female Tetranychus urticae (two-spotted spider mite). 43, 37-44, https://doi.org/10.1016/0048-3575(92)90017-T (1992).

42. Nguyen, A. H. et al. Loss of Arabidopsis 5' - $3^{\prime}$ Exoribonuclease AtXRN4 Function Enhances Heat Stress Tolerance of Plants Subjected to Severe Heat Stress. Plant Cell Physiol 56, 1762-1772, https://doi.org/10.1093/pcp/pcv096 (2015). 
43. Sako, K. et al. Proteomic Analysis of the 26S Proteasome Reveals Its Direct Interaction with Transit Peptides of Plastid Protein Precursors for Their Degradation. Journal of Proteome Research 13, 3223-3230, https://doi.org/10.1021/pr401245g (2014).

44. Yoshida, K., Noguchi, K., Motohashi, K. \& Hisabori, T. Systematic exploration of thioredoxin target proteins in plant mitochondria. Plant Cell Physiol 54, 875-892, https://doi.org/10.1093/pcp/pct037 (2013).

45. Miller, C. O. Cytokinin modification of mitochondrial function. Plant Physiol 69, 1274-1277, https://doi.org/10.1104/pp.69.6.1274 (1982).

46. Sue, M., Miyoshi, H. \& Iwamura, H. Specific interaction of cytokinins and their analogs with rotenone-sensitive internal NADH dehydrogenase in potato tuber mitochondria. Biosci Biotechnol Biochem 61, 1806-1809, https://doi.org/10.1271/bbb.61.1806 (1997).

47. Kanoh, N., Honda, K., Simizu, S., Muroi, M. \& Osada, H. Photo-cross-linked small-molecule affinity matrix for facilitating forward and reverse chemical genetics. Angew Chem Int Ed Engl 44, 3559-3562, https://doi.org/10.1002/anie.200462370 (2005).

\section{Acknowledgements}

We thank Dr. Takehiro Suzuki (RIKEN), and Dr. Naoshi Dohmae (RIKEN) for proteomic experiments. We also thank Edanz Group (www.edanzediting.com) for editing a draft of this manuscript. This project was financially supported by grants from RIKEN, the Japan Science and Technology Agency, the Core Research for Evolutional Science and Technology project (Grant Number JPMJCR13B4 to MS); the Ministry of Education, Culture, Sports, Science and Technology of Japan (KAKENHI Grant Number JP16H01476, JP18H04791 and JP18H04705 to MS, JP17H06412 to HO, JP18H05503 to YK, JP18H02140 to TN); and the Japan Society for the Promotion of Science (KAKENHI Grant Numbers JP16K18838 and JP18K14441 to K, Sako).

\section{Author contributions}

K., Sako and M.S. conceived the idea for the project. K., Sako, Y.F., T.N. and K.N. designed the experiments. K., Sako, Y.F., H.A., M.T., M.M. and K.N. performed the experiments. T.S., K., Shimizu, H.H., Y.K., K.H. and H.O. synthesized compounds and provided chemical library. A.M. and M.T. carried out the transcriptome analysis. K. Sako, Y.F., A.M., H.A., M.K. and M.S. analyzed the data. K., Sako, T.S. and M.S. wrote the manuscript.

\section{Competing interests}

The authors declare no competing interests.

\section{Additional information}

Supplementary information is available for this paper at https://doi.org/10.1038/s41598-020-65614-9.

Correspondence and requests for materials should be addressed to K.S. or M.S.

Reprints and permissions information is available at www.nature.com/reprints.

Publisher's note Springer Nature remains neutral with regard to jurisdictional claims in published maps and institutional affiliations.

(c) (i) Open Access This article is licensed under a Creative Commons Attribution 4.0 International License, which permits use, sharing, adaptation, distribution and reproduction in any medium or format, as long as you give appropriate credit to the original author(s) and the source, provide a link to the Creative Commons license, and indicate if changes were made. The images or other third party material in this article are included in the article's Creative Commons license, unless indicated otherwise in a credit line to the material. If material is not included in the article's Creative Commons license and your intended use is not permitted by statutory regulation or exceeds the permitted use, you will need to obtain permission directly from the copyright holder. To view a copy of this license, visit http://creativecommons.org/licenses/by/4.0/.

(c) The Author(s) 2020 